\title{
Enhancing the distance minimization methods of matrix updating within a homothetic paradigm
}

\author{
Vladimir Motorin ${ }^{*}$ (1)
}

\section{${ }^{*}$ Correspondence:}

motoriny@gmail.com; vmotorin@hse.ru

Laboratory for Research in Inflation and Growth, Expert Institute, National Research University Higher School of Economics,

Moscow, Russian Federation

\begin{abstract}
Matrix updating methods are used for constructing the target matrix with the prescribed row and column marginal totals that demonstrates the highest possible level of its structural similarity to initial matrix given. A concept of structural similarity has a vague framework that can be slightly refined under considering a particular case of strict proportionality between row and column marginal totals for target and initial matrices. Here the question arises: can we accept the initial matrix homothety as optimal solution for proportionality case of matrix-updating problem? In most practical situations, an affirmative answer to the question is almost obvious. It is natural to call this common notion by homothetic paradigm and to refer its checking as homothetic testing. Some well-known methods for matrix updating serve as an additional instrumental confirmation to validity of homothetic paradigm. It is shown that RAS method and Kuroda's method pass through the homothetic test successfully. Homothetic paradigm can be helpful for enhancing a collection of matrix updating methods based on constrained minimization of the distance functions. Main attention is paid to improving the methods with weighted squared differences (both regular and relative) as an objective function. As an instance of a incorrigible failure in the homothetic testing, the GRAS method for updating the economic matrices with some negative entries is analyzed in details. A collection of illustrative numerical examples and some recommendations for method's choice are given.
\end{abstract}

Keywords: Matrix updating methods, Homothetic paradigm and testing, RAS and Kuroda's methods, Kullback-Leibler divergence, Methods of weighted squared differences, GRAS method

JEL Classification: C61, C67

\section{An introduction to the matrix-updating problems}

A general problem of updating rectangular (or square) matrix can be formulated as follows. Let $\mathbf{A}$ be an initial matrix of dimension $N \times M$ with row and column marginal totals $\mathbf{u}_{\mathbf{A}}=\mathbf{A} \mathbf{e}_{M}, \mathbf{v}_{\mathbf{A}}^{\prime}=\mathbf{e}_{N}^{\prime} \mathbf{A}$ where $\mathbf{e}_{N}$ and $\mathbf{e}_{M}$ are $N \times 1$ and $M \times 1$ summation column vectors with unit elements. Further, let $\mathbf{u} \neq \mathbf{u}_{\mathbf{A}}$ and $\mathbf{v} \neq \mathbf{v}_{\mathbf{A}}$ be exogenous column vectors of dimension $N \times 1$ and $M \times 1$, respectively. The problem is to estimate a target matrix $\mathbf{X}$ of dimension $N \times M$ at the highest possible level of its structural similarity (or resemblance, likeness, closeness, etc.) to initial matrix A subject to $N+M$ equality constraints

(c) The Author(s) 2017. This article is distributed under the terms of the Creative Commons Attribution 4.0 International License (http://creativecommons.org/licenses/by/4.0/), which permits unrestricted use, distribution, and reproduction in any medium, provided you give appropriate credit to the original author(s) and the source, provide a link to the Creative Commons license, and indicate if changes were made. 


$$
\mathbf{X} \mathbf{e}_{M}=\mathbf{u}, \quad \mathbf{e}_{N}^{\prime} \mathbf{X}=\mathbf{v}^{\prime}
$$

and under the consistency condition

$$
\mathbf{e}_{N}^{\prime} \mathbf{u}=\mathbf{e}_{M}^{\prime} \mathbf{v}
$$

It is assumed that initial matrix A does not include any zero rows or zero columns, does not have less than $N+M$ nonzero elements, does not include any rows or columns with a unique nonzero element, and does not contain any pairs of rows and columns with four nonzero elements in the intersections. Otherwise, it is advisable to clean matrix A from those undesirable features before applying any matrix updating method in practice.

Clearly, the system of equations (1) is dependent at consistency condition (2) that provides an existence of target matrix $\mathbf{X}$. However, it is easy to show that any $N+M-1$ among $N+M$ constraints (1) are mutually independent. Furthermore, it is evident that any feasible solution of matrix-updating problem $\mathbf{X}$ can be simply transformed into another one by letting, e.g.,

$$
x_{n m}^{n e w}=x_{n m}+\varepsilon, \quad x_{n j}^{n e w}=x_{n j}-\varepsilon ; \quad x_{i m}^{n e w}=x_{i m}-\varepsilon, \quad x_{i j}^{n e w}=x_{i j}+\varepsilon
$$

where $\varepsilon$ is an arbitrary scalar, or

$$
\begin{aligned}
& x_{n m}^{\text {new }}=x_{n m}+\varepsilon, \quad x_{n j}^{n e w}=x_{n j}-\varepsilon / 2, \quad x_{n k}^{n e w}=x_{n k}-\varepsilon / 2 ; \quad x_{i m}^{\text {new }}=x_{i m}-\varepsilon, \\
& x_{i j}^{\text {new }}=x_{i j}+\varepsilon / 2, \quad x_{i k}^{n e w}=x_{i k}+\varepsilon / 2,
\end{aligned}
$$

and so on.

Thus, general problem of matrix updating with a great variety of feasible solutions significantly depends on a definition of the measure for structural similarity between initial and target matrices. Various definitions of this measure generate a great manifold of different methods and techniques for matrix updating. A quite common approach to define the similarity measure is to use some objective distance function $f(\mathbf{X}-\mathbf{A})$ to be minimized subject to linear constraints (1) under the consistency condition (2). The main purpose of the article is to enhance the methods of this kind by defining and applying a new algebraic concept named homothetic paradigm.

\section{A homothetic paradigm for the matrix updating methods}

A notion of structural similarity between initial and target matrices has a vague framework that can be slightly refined in an axiomatic manner. In this context, let us consider a particular case of strict proportionality between row and column marginal totals $\mathbf{u}=k \mathbf{u}_{\mathrm{A}}$ and $\mathbf{v}=k \mathbf{v}_{\mathbf{A}}$ for target and initial matrices with the same scalar factor $k$. Here the main question arises: can we accept the matrix homothety $\mathbf{X}=k \mathbf{A}$ as optimal solution for proportionality case of a general matrix-updating problem? At first sight this solution can be appreciated as rather logical and corresponding to famous principle of insufficient reason (also known in decision theory as Laplace criterion) because there is no way to explain convincingly that $\mathbf{X}$ must not be equal to $k \mathbf{A}$. Moreover, it allows preserving in $\mathrm{X}$ the same location of zeros as in the initial matrix. However, it is to be 
emphasized that the above question indeed seems neither simple nor evident, and its proposition cannot be proved formally.

Nevertheless, in most practical situations an affirmative answer to this question is almost obvious. In particular, as it is shown below, the well-known and widely used RAS and Kuroda's methods for matrix updating serve as an additional instrumental confirmation to such an answer. In this connection, we will call this rather common notion by homothetic paradigm and will refer examining the property "if $\mathbf{u}=k \mathbf{u}_{\mathrm{A}}$ and $\mathbf{v}=k \mathbf{v}_{\mathrm{A}}$ then $\mathbf{X}=k \mathbf{A}^{\prime \prime}$ as a homothetic test for the matrix updating methods. It is advisable to propose that a successful passing through homothetic test were to be appreciated as a positive feature of any matrix updating method.

\section{Homothetic testing of RAS method}

The key idea of the RAS method is triple factorization of target matrix

$$
\mathbf{X}=\mathbf{R A S}=\langle\mathbf{r}\rangle \mathbf{A}\langle\mathbf{s}\rangle=\hat{\mathbf{r}} \mathbf{A} \hat{\mathbf{s}}
$$

where $\mathbf{r}$ and $\mathbf{s}$ are unknown $N \times 1$ and $M \times 1$ column vectors. Here angled bracketing around vector's symbol or putting a "hat" over it denotes a diagonal matrix, with the vector on its main diagonal and zeros elsewhere (see Miller and Blair 2009, p. 697).

Putting (3) into (1), we have the system of nonlinear equations

$$
\hat{\mathbf{r}} \mathbf{A} \hat{\mathbf{s}} \mathbf{e}_{M}=\hat{\mathbf{r}} \mathbf{A} \hat{\mathbf{s}}=\langle\mathbf{A s}\rangle \mathbf{r}=\mathbf{u}, \quad \mathbf{e}_{N}^{\prime} \hat{\mathbf{r}} \mathbf{A} \hat{\mathbf{s}}=\mathbf{r}^{\prime} \mathbf{A} \hat{\mathbf{s}}=\mathbf{s}^{\prime}\left\langle\mathbf{A}^{\prime} \mathbf{r}\right\rangle=\mathbf{v}^{\prime} .
$$

Proper transformations of this system lead to the following pair of iterative processes:

$$
\begin{aligned}
& \mathbf{r}_{(i)}=\left\langle\mathbf{A}\left\langle\mathbf{A}^{\prime} \mathbf{r}_{(i-1)}\right\rangle^{-1} \mathbf{v}\right\rangle^{-1} \mathbf{u}, \quad i=1 \div I ; \quad \mathbf{s}_{(I)}=\left\langle\mathbf{A}^{\prime} \mathbf{r}_{(I)}\right\rangle^{-1} \mathbf{v} \\
& \mathbf{s}_{(j)}=\left\langle\mathbf{A}^{\prime}\left\langle\mathbf{A} \mathbf{s}_{(j-1)}\right\rangle^{-1} \mathbf{u}\right\rangle^{-1} \mathbf{v}, \quad j=1 \div J ; \quad \mathbf{r}_{(J)}=\left\langle\mathbf{A} \mathbf{s}_{(J)}\right\rangle^{-1} \mathbf{u}
\end{aligned}
$$

where $i$ and $j$ are iteration numbers, and the character " $\div$ " between the lower and upper bounds of index's changing range means that the index sequentially runs all integer values in the specified range.

As concerning a homothetic test for RAS method at $\mathbf{u}=k \mathbf{u}_{\mathrm{A}}$ and $\mathbf{v}=k \mathbf{v}_{\mathrm{A}}$, it can be easily shown that under starting condition $\mathbf{r}_{(0)}=\mathbf{e}_{N}$ or $\mathbf{s}_{(0)}=\mathbf{e}_{M}$ the RAS method iterative process (4) or (5) demonstrates one-step convergence to pair of vectors $\mathbf{r}=\mathbf{e}_{N}$, $\mathbf{s}=k \mathbf{e}_{M}$ or to $\mathbf{r}=k \mathbf{e}_{N}, \mathbf{s}=\mathbf{e}_{M}$, respectively. Hence, RAS algorithm's implementation gives $r_{n} s_{m}=k$ for any $n$ and $m, n=1 \div N, m=1 \div M$, from which $\mathbf{X}=\mathbf{r s}^{\prime} \circ \mathbf{A}=k \mathbf{A}$ where the character "o" denotes Hadamard's (element-wise) product of two matrices of the same dimension. Besides, it is easy to see that replacing the initial matrix $\mathbf{A}$ with its homothety $k A$ leaves the RAS method iterations (4) and (5) invariant. Thus, the RAS method passes through a homothetic test successfully.

For another formal proof of this fact, notice that the RAS method can be associated with a conditional minimization of nonnegative function called the Kullback-Leibler divergence (see Kullback and Leibler 1951; Kullback 1959) that is used for comparing "true" and "test" probability distributions. This function is sometimes called "information gain," albeit it measures an information loss from using a "test" distribution to 
approximate a "true" distribution. The Kullback-Leibler divergence actually expresses the difference between the cross-entropy of two distributions (namely, in order "true""test") and the entropy of "true" distribution.

Let the "true" distribution be $\mathbf{X} / x$, and let the "test" one be $\mathbf{A} / a$, where $a=\mathbf{e}_{N}^{\prime} \mathbf{A} \mathbf{e}_{M}$ and $x=\mathbf{e}_{N}^{\prime} \mathbf{X} \mathbf{e}_{M}=\mathbf{e}_{N}^{\prime} \mathbf{u}=\mathbf{e}_{M}^{\prime} \mathbf{v}$. (So all elements of $\mathbf{X}$ and $\mathbf{A}$ are implied to be nonnegative.) In these denotations the Kullback-Leibler divergence can be written as

$$
f_{K L}(\mathbf{X} / x ; \mathbf{A} / a)=\sum_{n=1}^{N} \sum_{m=1}^{M} \frac{x_{n m}}{x} \ln \left(\frac{a}{x} \frac{x_{n m}}{a_{n m}}\right)=\frac{1}{x} f_{K L}(\mathbf{X} ; \mathbf{A})+\ln \frac{a}{x}
$$

where $f_{K L}(\mathbf{X} ; \mathbf{A})$ is corresponding Kullback-Leibler function for nonnormalized data. Note that values of $f_{K L}(\mathbf{X} / x ; \mathbf{A} / a)$ are dimensionless quantities, whereas $f_{K L}(\mathbf{X} ; \mathbf{A})$ has dimension of $x$ (or of X's and A's entries).

The function $f_{K L}(\mathbf{X} ; \mathbf{A})$ for nonnormalized data often serves as an objective function in mathematical programming formulation of the RAS method instead of function (6), e.g., in Appendix 7.1 "RAS as a Solution to the Constrained Minimum Information Distance Problem" to Miller and Blair (2009). It becomes possible due to the linear dependency (6) between $f_{K L}(\mathbf{X} / x ; \mathbf{A} / a)$ and $f_{K L}(\mathbf{X} ; \mathbf{A})$ with fixed and known coefficients, so that a minimum of both functions is achieved at the same point. Nevertheless, the Kullback-Leibler function for nonnormalized data cannot be classified as "information distance" at least from the viewpoint of dimensional analysis. (To be fully fair, the Kullback-Leibler divergence (6) is not a distance function really because the symmetry and subadditivity (triangle inequality) conditions do not hold for it.)

Consider the conditional minimization problem with objective function (6) for normalized data and linear constraints (1) under the consistency requirement $\mathbf{e}_{N}^{\prime} \mathbf{u}=\mathbf{e}_{M}^{\prime} \mathbf{v}=\mathbf{e}_{N}^{\prime} \mathbf{X} \mathbf{e}_{M}=x$.

The first partial derivatives of Lagrangian function for this problem with respect to $x_{n m}$ are

$$
\frac{\partial L_{K L}}{\partial x_{n m}}=\frac{1}{x}\left(\ln x_{n m}+1-\ln a_{n m}\right)-\lambda_{n}-\mu_{m}=0, \quad n=1 \div N, m=1 \div M,
$$

from which it is easy to obtain the RAS triple factorization of target matrix (3) as follows:

$$
x_{n m}=a_{n m} e^{x\left(\lambda_{n}+\mu_{m}\right)-1}=e^{x \lambda_{n}-1 / 2} a_{n m} e^{x \mu_{m}-1 / 2}=r_{n} a_{n m} s_{m}
$$

where $\lambda$ and $\mu$ are the column vectors of Lagrange multipliers with dimensions $N \times 1$ and $M \times 1$, respectively, and $e$ is the base of natural logarithms.

Thus, the RAS method's logical emanation from the Kullback-Leibler divergence minimization approach is proved.

Finally, it is easy to see that in homothetic testing with $\mathbf{X}=k \mathbf{A}$ the nonnegative function (6) for normalized data reaches its global (or absolute) minimum value, since

$$
f_{K L}(k \mathbf{A} / k a ; \mathbf{A} / a)=\sum_{n=1}^{N} \sum_{m=1}^{M} \frac{k a_{n m}}{k a} \ln \left(\frac{a}{k a} \frac{k a_{n m}}{a_{n m}}\right)=\frac{k a}{k a} \ln (1)=0,
$$


although function $f_{K L}(\mathbf{X} ; \mathbf{A})$ for nonnormalized data does not allow to consider the matrices $\mathbf{X}=k \mathbf{A}$ and $\mathbf{A}$ as indiscernibles, namely

$$
f_{K L}(k \mathbf{A} ; \mathbf{A})=\sum_{n=1}^{N} \sum_{m=1}^{M} k a_{n m} \ln \left(\frac{k a_{n m}}{a_{n m}}\right)=k a \cdot \ln (k) \neq 0 .
$$

It means that, from viewpoint of the Kullback-Leibler divergence minimization approach together with the RAS method, the matrix homothety $\mathbf{X}=k \mathbf{A}$ can be considered as optimal solution for proportionality case of a general matrix-updating problem.

\section{Homothetic testing of Kuroda's method}

Kuroda (1988) proposed an original method for matrix updating that comes to constrained minimization of the twofold-weighted quadratic objective function

$$
f_{K}(\mathbf{X} ; \mathbf{A})=\frac{1}{2} \sum_{n=1}^{N} \sum_{m=1}^{M} w_{N M}^{1}\left(\frac{x_{n m}}{u_{n}}-\frac{a_{n m}}{u_{n}^{\mathbf{A}}}\right)^{2}+\frac{1}{2} \sum_{n=1}^{N} \sum_{m=1}^{M} w_{N M}^{2}\left(\frac{x_{n m}}{v_{m}}-\frac{a_{n m}}{v_{m}^{\mathbf{A}}}\right)^{2},
$$

which can be rewritten in matrix form as

$$
f_{K}\left(\mathbf{x}_{\mathbf{u}}, \mathbf{x}_{\mathbf{v}}\right)=\frac{1}{2} \mathbf{x}_{\mathbf{u}}^{\prime} \mathbf{W}_{1} \mathbf{x}_{\mathbf{u}}+\frac{1}{2} \mathbf{x}_{\mathbf{v}}^{\prime} \mathbf{W}_{2} \mathbf{x}_{\mathbf{v}}
$$

where $\mathbf{W}_{1}$ and $\mathbf{W}_{2}$ are the nonsingular diagonal matrices of order $N M$ with the relative reliability or relative confidence factors (weights). Here the NM-dimensional column vectors $\mathbf{x}_{\mathbf{u}}$ and $\mathbf{x}_{\mathbf{v}}$ are defined through applying the vectorization operator "vec," which transforms a matrix into a vector by stacking the columns of the matrix one underneath the other (see, e.g., Magnus and Neudecker 2007), as follows:

$$
\mathbf{x}_{\mathbf{u}}=\operatorname{vec}\left(\hat{\mathbf{u}}^{-1} \mathbf{X}-\hat{\mathbf{u}}_{\mathbf{A}}^{-1} \mathbf{A}\right)=\mathbf{U} \mathbf{x}-\mathbf{U}_{\mathbf{A}} \mathbf{a}, \quad \mathbf{x}_{\mathbf{v}}=\operatorname{vec}\left(\mathbf{X} \hat{\mathbf{v}}^{-1}-\mathbf{A} \hat{\mathbf{v}}_{\mathbf{A}}^{-1}\right)=\mathbf{V} \mathbf{x}-\mathbf{V}_{\mathbf{A}} \mathbf{a},
$$

where

$$
\begin{aligned}
& \mathbf{a}=\operatorname{vec} \mathbf{A}, \quad \mathbf{U}_{\mathbf{A}}=\mathbf{E}_{M} \otimes \hat{\mathbf{u}}_{\mathbf{A}}^{-1}, \quad \mathbf{V}_{\mathbf{A}}=\hat{\mathbf{v}}_{\mathbf{A}}^{-1} \otimes \mathbf{E}_{N} \\
& \mathbf{x}=\operatorname{vec} \mathbf{X}, \quad \mathbf{U}=\mathbf{E}_{M} \otimes \hat{\mathbf{u}}^{-1}, \quad \mathbf{V}=\hat{\mathbf{v}}^{-1} \otimes \mathbf{E}_{N},
\end{aligned}
$$

$\mathbf{E}_{M}$ is an identity matrix of order $M$, and the character " $\otimes$ " denotes the Kronecker product of two matrices.

Within a homothetic test for Kuroda's method, the row and column marginal totals for target matrix are $\mathbf{u}=k \mathbf{u}_{\mathbf{A}}$ and $\mathbf{v}=k \mathbf{v}_{\mathbf{A}}$, hence

$$
\mathbf{x}_{\mathbf{u}}=\mathbf{U} \mathbf{x}-\mathbf{U}_{\mathrm{A}} \mathbf{a}=\mathbf{U}_{\mathbf{A}}\left(\frac{1}{k} \mathbf{x}-\mathbf{a}\right), \quad \mathbf{x}_{\mathbf{v}}=\mathbf{V} \mathbf{x}-\mathbf{V}_{\mathbf{A}} \mathbf{a}=\mathbf{V}_{\mathbf{A}}\left(\frac{1}{k} \mathbf{x}-\mathbf{a}\right) .
$$

Therefore, at $\mathbf{x}=k \mathbf{a}$ the vectors $\mathbf{x}_{\mathbf{u}}$ and $\mathbf{x}_{\mathbf{v}}$ vanish both, and the quadratic function (7) reaches its global minimum value equal to zero. It means that from viewpoint of Kuroda's method, the matrix homothety $\mathbf{X}=k \mathbf{A}$ provides the optimal solution for a general problem of matrix updating in a case of strict proportionality between row and column 
marginal totals for target and initial matrices. Thus, Kuroda's method passes through a homothetic test successfully as well as RAS method.

\section{Homothetic test's failure: the method of weighted squared differences (WSD)}

A quite common approach to define a measure of the structural similarity between initial and target matrices is to use some matrix norm for the difference $\mathbf{X}-\mathbf{A}$ to be minimized subject to linear constraints (1) under the consistency condition (one can find the proper reviews, e.g., in Miller and Blair 2009; Temurshoev et al. 2011), so that the optimal solution can be represented as $\mathbf{X}^{*}=\arg \min _{\mathbf{X}}\|\mathbf{X}-\mathbf{A}\|$, or in equivalent vector form as $\mathbf{x}^{*}=\arg \min _{\mathbf{x}}|\mathbf{x}-\mathbf{a}|$ where $\mathbf{a}=\operatorname{vec} \mathbf{A}$ and $\mathbf{x}=\operatorname{vec} \mathbf{X}$, as earlier.

For instance, the choice of Frobenius matrix norm (and compatible Euclidean vector norm) leads to the constrained minimization of weighted quadratic objective function

$$
f_{W S D}(\mathbf{x} ; \mathbf{a})=\sum_{n=1}^{N} \sum_{m=1}^{M} w_{n m}\left(x_{n m}-a_{n m}\right)^{2}=(\mathbf{x}-\mathbf{a})^{\prime} \mathbf{W}(\mathbf{x}-\mathbf{a})
$$

where $\mathbf{W}$ is a nonsingular diagonal matrix of order $N M$ with the relative reliability or relative confidence coefficients. (Usually the elements of matrix $\mathbf{W}$ is assumed to be normalized by multiplying it on a proper factor, i.e., $\mathbf{e}_{N M}^{\prime} \mathbf{W} \mathbf{e}_{N M}=1$.) To complete a formulation of the constrained minimization problem for the WSD method, one needs to rewrite left-hand sides of the constraints (1) in vector denotations. Introducing the $N \times N M$ matrix $\mathbf{G}=\mathbf{e}_{M}^{\prime} \otimes \mathbf{E}_{N}$, which consists of $M$ identity matrix $\mathbf{E}_{N}$ located horizontally, and the $M \times N M$ matrix $\mathbf{H}=\mathbf{E}_{M} \otimes \mathbf{e}_{N}^{\prime}$, which is $N$-fold successive replication of each column from identity matrix $\mathbf{E}_{M}$, we have

$$
\mathbf{X} \mathbf{e}_{M}=\mathbf{G x}=\mathbf{u}, \quad \mathbf{X}^{\prime} \mathbf{e}_{N}=\mathbf{H} \mathbf{x}=\mathbf{v} .
$$

Notice that each column of $\mathbf{G}$ and $\mathbf{H}$ includes exactly one nonzero (unit) element such that $\mathbf{e}_{N}^{\prime} \mathbf{G}=\mathbf{e}_{M}^{\prime} \mathbf{H}=\mathbf{e}_{N M}^{\prime}$.

The Lagrangian function for problem of minimizing the objective function (8) subject to linear constraints (9) is just

$$
L_{W S D}(\mathbf{x}, \boldsymbol{\lambda}, \boldsymbol{\mu})=(\mathbf{x}-\mathbf{a})^{\prime} \mathbf{W}(\mathbf{x}-\mathbf{a})-\lambda^{\prime}(\mathbf{G} \mathbf{x}-\mathbf{u})-\boldsymbol{\mu}^{\prime}(\mathbf{H} \mathbf{x}-\mathbf{v})
$$

where $\lambda$ and $\mu$ are the column vectors of Lagrange multipliers, as earlier. Taking the first partial derivatives of this function with respect to $\mathbf{x}, \boldsymbol{\lambda}$ and $\boldsymbol{\mu}$ gives

$$
2 \mathbf{W}(\mathbf{x}-\mathbf{a})-\mathbf{G}^{\prime} \lambda-\mathbf{H}^{\prime} \boldsymbol{\mu}=\mathbf{0}_{N M}, \quad \mathbf{G x}-\mathbf{u}=\mathbf{0}_{N}, \quad \mathbf{H x}-\mathbf{v}=\mathbf{0}_{M} .
$$

Expressing $\mathbf{x}$ from first equation of this system, we obtain the problem solution as a function of Lagrange multipliers, namely

$$
\mathbf{x}=\mathbf{a}+\frac{1}{2} \mathbf{W}^{-1}\left(\mathbf{G}^{\prime} \boldsymbol{\lambda}+\mathbf{H}^{\prime} \boldsymbol{\mu}\right) .
$$

Inserting (10) into the second and third equations leads to the following system of equations with $\lambda$ and $\mu$ as unknowns: 


$$
\begin{aligned}
& \mathbf{G W}^{-1} \mathbf{G}^{\prime} \boldsymbol{\lambda}+\mathbf{G W}^{-1} \mathbf{H}^{\prime} \boldsymbol{\mu}=2(\mathbf{u}-\mathbf{G a}), \\
& \mathbf{H} \mathbf{W}^{-1} \mathbf{G}^{\prime} \boldsymbol{\lambda}+\mathbf{H W}^{-1} \mathbf{H}^{\prime} \boldsymbol{\mu}=2(\mathbf{v}-\mathbf{H a})
\end{aligned}
$$

where $\mathbf{G a}=\mathbf{u}_{\mathrm{A}}$ and $\mathbf{H a}=\mathbf{v}_{\mathrm{A}}$.

Note that the main matrix of the system (11) is symmetric. Moreover, it is easy to see that $\mathbf{G} \mathbf{W}^{-1} \mathbf{G}^{\prime} \mathbf{e}_{N}-\mathbf{G} \mathbf{W}^{-1} \mathbf{H}^{\prime} \mathbf{e}_{M}=\mathbf{0}_{N}$ and $\mathbf{H} \mathbf{W}^{-1} \mathbf{G}^{\prime} \mathbf{e}_{N}-\mathbf{H} \mathbf{W}^{-1} \mathbf{H}^{\prime} \mathbf{e}_{M}=\mathbf{0}_{M}$ because of $\mathbf{G}^{\prime} \mathbf{e}_{N}=\mathbf{H}^{\prime} \mathbf{e}_{M}=\mathbf{e}_{N M}$, i.e., the matrix of the system (11) has the linearly dependent columns and so is singular. Thus, the general solution to corresponding homogeneous system (11) is $\lambda^{(0)}=c \mathbf{e}_{N}, \boldsymbol{\mu}^{(0)}=-c \mathbf{e}_{M}$ with any scalar constant $c$.

Since a general solution to nonhomogeneous linear system equals the sum of a general solution to corresponding homogeneous system and any particular solution to nonhomogeneous system, let $\lambda=\lambda^{(0)}+\lambda^{(1)}$ and $\mu=\mu^{(0)}+\mu^{(1)}$, where the pair $\lambda^{(1)}, \mu^{(1)}$ is a particular solution to system (11). Putting these formulas into round-bracketed expression in the right-hand side of (10) gives

$$
\begin{aligned}
\mathbf{G}^{\prime} \boldsymbol{\lambda} & +\mathbf{H}^{\prime} \boldsymbol{\mu}=\mathbf{G}^{\prime}\left(c \mathbf{e}_{N}+\lambda^{(1)}\right)+\mathbf{H}^{\prime}\left(-c \mathbf{e}_{M}+\boldsymbol{\mu}^{(1)}\right) \\
& =c \mathbf{e}_{N M}-c \mathbf{e}_{N M}+\mathbf{G}^{\prime} \boldsymbol{\lambda}^{(1)}+\mathbf{H}^{\prime} \boldsymbol{\mu}^{(1)}=\mathbf{G}^{\prime} \boldsymbol{\lambda}^{(1)}+\mathbf{H}^{\prime} \boldsymbol{\mu}^{(1)},
\end{aligned}
$$

i.e., the optimal solution of minimization problem (8), (9) depends only on a particular solution to nonhomogeneous system (11).

As noted above, any $N+M-1$ among $N+M$ constraints (9) are mutually independent while $\mathbf{e}_{N}^{\prime} \mathbf{u}=\mathbf{e}_{M}^{\prime} \mathbf{v}$. Therefore, the Lagrange multipliers $\lambda$ and $\boldsymbol{\mu}$ can be determined from reduced system of linear equations (11) with any one of them eliminated (of course, with setting a corresponding multiplier equal to zero).

For homothetic testing the WSD method, suppose that $\mathbf{x}=k \mathbf{a}$. Inserting this homothety into the solution (10) leads to the condition

$$
\mathbf{G}^{\prime} \lambda+\mathbf{H}^{\prime} \boldsymbol{\mu}=2(k-1) \mathbf{W} \mathbf{a}
$$

which is not met at $N+M>3$ because it actually represents a generally inconsistent system of $N M$ linear equations with $N+M-1<N M$ unknown Lagrange multipliers. (Recall that one of them equals zero.) Hence, the vector $\mathbf{x}=k \mathbf{a}$ cannot be an optimal solution of minimization problem (8), (9) at $\mathbf{u}=k \mathbf{u}_{\mathrm{A}}$ and $\mathbf{v}=k \mathbf{v}_{\mathbf{A}}$.

Thus, WSD method does not pass through a homothetic test in contrast to RAS and Kuroda's method.

\section{Applying homothetic paradigm for improving the WSD method}

Acceptance of the matrix $\mathbf{X}=k \mathbf{A}$ as optimal solution for proportionality case of a general matrix updating problem leads to establishing the fact that all matrices from homothetic family $k \mathbf{A}$ demonstrate an excellent structural similarity between each other. The homothetic paradigm can be helpful for enhancing a collection of matrix updating methods based on constrained minimization of the distance (or quasi-distance) functions.

Within homothetic paradigm, we can set a goal to dispose the target matrix as close as possible not to initial matrix $\mathbf{A}$, but to its homothetic family $k \mathbf{A}$. As a result, the optimal solution becomes $\left(\mathbf{X}^{*}, k^{*}\right)=\arg \min _{\mathbf{X}, k}\|\mathbf{X}-k \mathbf{A}\|$, or in equivalent vector form 
$\left(\mathbf{x}^{*}, k^{*}\right)=\arg \min _{\mathbf{x}, k}|\mathbf{x}-k \mathbf{a}|$, and, clearly, it cannot be "worse" (in terms of the certain matrix/vector norm chosen) than the original one.

As it is shown below, transition from $|\mathbf{x}-\mathbf{a}|$ to $|\mathbf{x}-k \mathbf{a}|$ leads to an idea of orthogonal projecting an unknown target vector $\mathbf{x}$ onto the homothetic ray $k \mathbf{a}$ in $N M$-dimensional vector space with a scalar product operation.

In the case of WSD method, the improved version of weighted quadratic objective function (8) can be written as

$$
f_{i W S D}(\mathbf{x}, l ; \mathbf{a})=\sum_{n=1}^{N} \sum_{m=1}^{M} w_{n m}\left(x_{n m}-l a_{n m}\right)^{2}=(\mathbf{x}-l \mathbf{a})^{\prime} \mathbf{W}(\mathbf{x}-l \mathbf{a})
$$

where $l$ is an additional scalar variable.

The Lagrangian function for problem of minimizing the objective function (13) subject to linear constraints (9) becomes

$$
L_{i W S D}(\mathbf{x}, l, \boldsymbol{\lambda}, \boldsymbol{\mu})=(\mathbf{x}-l \mathbf{a})^{\prime} \mathbf{W}(\mathbf{x}-l \mathbf{a})-\lambda^{\prime}(\mathbf{G x}-\mathbf{u})-\boldsymbol{\mu}^{\prime}(\mathbf{H} \mathbf{x}-\mathbf{v})
$$

where, as earlier, $\lambda$ and $\mu$ are the column vectors of Lagrange multipliers. Taking the first partial derivatives of this function with respect to vector $\mathbf{x}$ and scalar $l$ gives the following system of $N M+1$ equations:

$$
2 \mathbf{W}(\mathbf{x}-l \mathbf{a})-\mathbf{G}^{\prime} \lambda-\mathbf{H}^{\prime} \boldsymbol{\mu}=\mathbf{0}_{N M}, \quad \mathbf{a}^{\prime} \mathbf{W}(\mathbf{x}-l \mathbf{a})=0
$$

where the second equation is being interpreted as an orthogonality condition for vectors $\mathbf{x}-l \mathbf{a}$ and $\mathbf{a}$.

Expressing $\mathbf{x}$ from first equation of this system, we obtain the solution of problem (13), (9) as a function of Lagrange multipliers, namely

$$
\mathbf{x}=l \mathbf{a}+\frac{1}{2} \mathbf{W}^{-1}\left(\mathbf{G}^{\prime} \boldsymbol{\lambda}+\mathbf{H}^{\prime} \boldsymbol{\mu}\right) .
$$

Inserting (14) into second equation of above system and into the pair of constraint equations (9) leads to the following system of $1+N+M$ equations with scalar $l$ and vectors $\lambda, \mu$ as unknown variables:

$$
\begin{aligned}
& \mathbf{a}^{\prime} \mathbf{G}^{\prime} \boldsymbol{\lambda}+\mathbf{a}^{\prime} \mathbf{H}^{\prime} \boldsymbol{\mu}=0, \\
& 2 l \mathbf{G a}+\mathbf{G} \mathbf{W}^{-1} \mathbf{G}^{\prime} \boldsymbol{\lambda}+\mathbf{G} \mathbf{W}^{-1} \mathbf{H}^{\prime} \boldsymbol{\mu}=2 \mathbf{u}, \\
& 2 l \mathbf{H a}+\mathbf{H} \mathbf{W}^{-1} \mathbf{G}^{\prime} \boldsymbol{\lambda}+\mathbf{H} \mathbf{W}^{-1} \mathbf{H}^{\prime} \boldsymbol{\mu}=2 \mathbf{v}
\end{aligned}
$$

where $\mathbf{G a}=\mathbf{u}_{\mathbf{A}}$ and $\mathbf{H a}=\mathbf{v}_{\mathbf{A}}$. Since any $N+M-1$ among $N+M$ constraints (9) are mutually independent while $\mathbf{e}_{N}^{\prime} \mathbf{u}=\mathbf{e}_{M}^{\prime} \mathbf{v}$, the scalar $l$ and Lagrange multipliers $\lambda, \mu$ can be determined from reduced system of linear equations (15) with any one of them eliminated (except the first equation) with setting a corresponding multiplier equal to zero.

Putting the improved WSD method to homothetic test, suppose that $\mathbf{x}=k \mathbf{a}$. Inserting this formula into the orthogonality condition gives $(k-l) \mathbf{a}^{\prime} \mathbf{W} \mathbf{a}=0$ from which $l^{*}=k$. In turn, substituting the homothety into (14) leads to the homogeneous system

$$
\mathbf{G}^{\prime} \boldsymbol{\lambda}+\mathbf{H}^{\prime} \boldsymbol{\mu}=\mathbf{0}_{N M}
$$


that obviously has the solution $\lambda^{*}=c \mathbf{e}_{N}, \mu^{*}=-c \mathbf{e}_{M}$ where $c$ is an arbitrary scalar (see Sect. 5). Further, at $\mathbf{u}=k \mathbf{u}_{\mathrm{A}}, \mathbf{v}=k \mathbf{v}_{\mathrm{A}}$ and $l^{*}=k$ the linear equations (15) are also being transformed to the homogeneous system

$$
\begin{aligned}
& \mathbf{a}^{\prime}\left(\mathbf{G}^{\prime} \boldsymbol{\lambda}+\mathbf{H}^{\prime} \boldsymbol{\mu}\right)=0, \\
& \mathbf{G} \mathbf{W}^{-1}\left(\mathbf{G}^{\prime} \boldsymbol{\lambda}+\mathbf{H}^{\prime} \boldsymbol{\mu}\right)=\mathbf{0}_{N}, \\
& \mathbf{H} \mathbf{W}^{-1}\left(\mathbf{G}^{\prime} \boldsymbol{\lambda}+\mathbf{H}^{\prime} \boldsymbol{\mu}\right)=\mathbf{0}_{M}
\end{aligned}
$$

with the same simple solution. Therefore, the vectors $\mathbf{x}^{*}=k \mathbf{a}, \lambda^{*}=c \mathbf{e}_{N}, \boldsymbol{\mu}^{*}=-c \mathbf{e}_{M}$ bring the optimal solution to the problem of minimizing the objective function (13) subject to linear constraints (9) at $\mathbf{u}=k \mathbf{u}_{\mathrm{A}}$ and $\mathbf{v}=k \mathbf{v}_{\mathrm{A}}$.

Thus, the improved WSD method passes through a homothetic test successfully as well as RAS and Kuroda's method (and in contrast to WSD method).

\section{Analyzing and improving the method of weighted squared relative differences (WSRD)}

To make the minimization problems (8), (9) and (13), (9) independent on scale of initial data, it is expedient to let

$$
\mathbf{x}=\hat{\mathbf{a} q}
$$

where $\mathbf{q}$ is $N M$-dimensional column vector of unknown relative coefficients, and then to introduce into consideration the relative distance functions, namely $\left|\mathbf{q}-\mathbf{e}_{N M}\right|$ instead of $|\mathbf{x}-\mathbf{a}|$ for WSD method and $\left|\mathbf{q}-k \mathbf{e}_{N M}\right|$ instead of $|\mathbf{x}-k \mathbf{a}|$ for improved WSD method. Here the transition from $\left|\mathbf{q}-\mathbf{e}_{N M}\right|$ to $\left|\mathbf{q}-k \mathbf{e}_{N M}\right|$, as it is shown below, leads to an idea of orthogonal projecting an unknown target vector $\mathbf{q}$ onto the homothetic ray $k \mathbf{e}_{N M}$ in $N M$-dimensional vector space with a scalar product operation. Notice that Eq. (16) cannot be resolved with respect to $\mathbf{q}$ if the initial matrix $\mathbf{A}$ contains at least one zero entry.

In transition from WSD to WSRD method, the quadratic objective function (8) becomes

$$
f_{W S R D}\left(\mathbf{q} ; \mathbf{e}_{N M}\right)=\sum_{n=1}^{N} \sum_{m=1}^{M} w_{n m}\left(q_{n m}-e_{n m}\right)^{2}=\left(\mathbf{q}-\mathbf{e}_{N M}\right)^{\prime} \mathbf{W}\left(\mathbf{q}-\mathbf{e}_{N M}\right)
$$

whereas the improved objective function (13) is being transformed to

$$
f_{i W S R D}\left(\mathbf{q}, l ; \mathbf{e}_{N M}\right)=\sum_{n=1}^{N} \sum_{m=1}^{M} w_{n m}\left(q_{n m}-l e_{n m}\right)^{2}=\left(\mathbf{q}-l \mathbf{e}_{N M}\right)^{\prime} \mathbf{W}\left(\mathbf{q}-l \mathbf{e}_{N M}\right),
$$

and, finally, inserting (16) into the linear constraints (9) leads to the following modified constraints:

$$
\mathbf{G x}=\mathbf{G a ̂ q}=\mathbf{u}, \quad \mathbf{H x}=\mathbf{H a ̂ q}=\mathbf{v} .
$$

The objective function (17) was first proposed by Harthoorn and van Dalen (1987) with the relative confidence coefficients matrix factorized as $\mathbf{W}=\hat{\mathbf{a}} \hat{\mathbf{w}}^{-1} \hat{\mathbf{a}}$ where $\mathbf{w}$ is 
exogenous vector of reciprocal weights for the elements of initial vector a. The Lagrangian function for problem of minimizing the objective function (17) subject to linear constraints (19) is just

$$
L_{W S R D}(\mathbf{q} ; \boldsymbol{\lambda}, \boldsymbol{\mu})=\left(\mathbf{q}-\mathbf{e}_{N M}\right)^{\prime} \mathbf{W}\left(\mathbf{q}-\mathbf{e}_{N M}\right)-\lambda^{\prime}(\mathbf{G a ̂ q}-\mathbf{u})-\boldsymbol{\mu}^{\prime}(\mathbf{H a ̂ q}-\mathbf{v}),
$$

where $\lambda$ and $\mu$ are the column vectors of Lagrange multipliers, as earlier.

By analogy with derivation of (10) and (11) in Sect. 5, we obtain

$$
\mathbf{q}=\mathbf{e}_{N M}+\frac{1}{2} \mathbf{W}^{-1} \hat{\mathbf{a}}\left(\mathbf{G}^{\prime} \boldsymbol{\lambda}+\mathbf{H}^{\prime} \boldsymbol{\mu}\right)
$$

and

$$
\begin{aligned}
& \mathbf{G a ̂} W^{-1} \hat{\mathbf{a}} \mathbf{G}^{\prime} \boldsymbol{\lambda}+\mathbf{G} \mathbf{a} \mathbf{W}^{-1} \hat{\mathbf{a}} \mathbf{H}^{\prime} \boldsymbol{\mu}=2(\mathbf{u}-\mathbf{G a}), \\
& \mathbf{H a ̂} \mathbf{W}^{-1} \hat{\mathbf{a}} \mathbf{G}^{\prime} \boldsymbol{\lambda}+\mathbf{H a} \mathbf{a} W^{-1} \hat{\mathbf{a}} \mathbf{H}^{\prime} \boldsymbol{\mu}=2(\mathbf{v}-\mathbf{H a})
\end{aligned}
$$

where, as earlier, $\mathbf{G a}=\mathbf{u}_{\mathbf{A}}$ and $\mathbf{H a}=\mathbf{v}_{\mathbf{A}}$. It is easy to see that the system of linear equations (21) is a degenerate one, resembling (11), and its corresponding homogeneous system also has the general solution $\lambda^{(0)}=c \mathbf{e}_{N}, \boldsymbol{\mu}^{(0)}=-c \mathbf{e}_{M}$ with any scalar constant $c$. Since any $N+M-1$ among $N+M$ constraints (19) are mutually independent while $\mathbf{e}_{N}^{\prime} \mathbf{u}=\mathbf{e}_{M}^{\prime} \mathbf{v}$, the Lagrange multipliers $\lambda$ and $\mu$ can be determined from reduced system of linear equations (21) with any one of them eliminated with setting a corresponding multiplier equal to zero.

For homothetic testing the WSRD method, suppose that $\mathbf{q}=k \mathbf{e}_{N M}$. Inserting this homothety into the solution (20) leads to the condition (compare with condition (12) in Sect. 5)

$$
\hat{\mathbf{a}}\left(\mathbf{G}^{\prime} \boldsymbol{\lambda}+\mathbf{H}^{\prime} \boldsymbol{\mu}\right)=2(k-1) \mathbf{W} \mathbf{e}_{N M}
$$

which is not met at $N+M>3$ because it actually represents a generally inconsistent system of $N M$ linear equations with $N+M-1<N M$ unknown Lagrange multipliers. (One of them equals zero because the matrix of system (21) has nonfull rank.) Hence, the vector $\mathbf{q}=k \mathbf{e}_{N M}$ cannot be an optimal solution of minimization problem (17), (19) at $\mathbf{u}=k \mathbf{u}_{\mathrm{A}}$ and $\mathbf{v}=k \mathbf{v}_{\mathrm{A}}$. Thus, WSRD method does not pass through a homothetic test as well as WSD method.

The objective function (18) demonstrates a result of applying a homothetic paradigm to the objective function of WSRD method (17). The Lagrangian function for problem of minimizing the improved objective function (18) subject to linear constraints (19) is just

$$
L_{i W S R D}(\mathbf{q}, l ; \boldsymbol{\lambda}, \boldsymbol{\mu})=\left(\mathbf{q}-l \mathbf{e}_{N M}\right)^{\prime} \mathbf{W}\left(\mathbf{q}-l \mathbf{e}_{N M}\right)-\lambda^{\prime}(\mathbf{G a ̂ q}-\mathbf{u})-\boldsymbol{\mu}^{\prime}(\mathbf{H a ̂ q}-\mathbf{v}),
$$

where $\lambda$ and $\mu$ are the column vectors of Lagrange multipliers, and $l$ is an additional scalar variable.

Taking the first partial derivatives of this function with respect to vector $\mathbf{q}$ and scalar $l$ gives the following system of $N M+1$ equations:

$$
2 \mathbf{W}\left(\mathbf{q}-l \mathbf{e}_{N M}\right)-\hat{\mathbf{a}} \mathbf{G}^{\prime} \lambda-\hat{\mathbf{a}} \mathbf{H}^{\prime} \boldsymbol{\mu}=\mathbf{0}_{N M}, \quad \mathbf{e}_{N M}^{\prime} \mathbf{W}\left(\mathbf{q}-l \mathbf{e}_{N M}\right)=0
$$


where the second equation of the system expresses an orthogonality condition for vectors $\mathbf{q}-l \mathbf{e}_{N M}$ and $\mathbf{e}_{N M}$.

By analogy with derivation of (14) and (15) in Sect. 6, we obtain

$$
\mathbf{q}=l \mathbf{e}_{N M}+\frac{1}{2} \mathbf{W}^{-1} \hat{\mathbf{a}}\left(\mathbf{G}^{\prime} \boldsymbol{\lambda}+\mathbf{H}^{\prime} \boldsymbol{\mu}\right)
$$

and

$$
\begin{aligned}
& \mathbf{a}^{\prime} \mathbf{G}^{\prime} \lambda+\mathbf{a}^{\prime} \mathbf{H}^{\prime} \boldsymbol{\mu}=0, \\
& 2 l \mathbf{G a}+\mathbf{G a ̂}^{-1} \hat{\mathbf{a}} \mathbf{G}^{\prime} \boldsymbol{\lambda}+\mathbf{G a ̂ W}^{-1} \hat{\mathbf{a}} \mathbf{H}^{\prime} \boldsymbol{\mu}=2 \mathbf{u}, \\
& 2 l \mathbf{H a}+\mathbf{H a} \mathbf{W}^{-1} \hat{\mathbf{a}} \mathbf{G}^{\prime} \boldsymbol{\lambda}+\mathbf{H a ̂}^{-1} \hat{\mathbf{a}} \mathbf{H}^{\prime} \boldsymbol{\mu}=2 \mathbf{v}
\end{aligned}
$$

where $\mathbf{G a}=\mathbf{u}_{\mathbf{A}}$ and $\mathbf{H a}=\mathbf{v}_{\mathbf{A}}$. Since any $N+M-1$ among $N+M$ constraints (19) are mutually independent while $\mathbf{e}_{N}^{\prime} \mathbf{u}=\mathbf{e}_{M}^{\prime} \mathbf{v}$, the scalar $l$ and Lagrange multipliers $\boldsymbol{\lambda}, \boldsymbol{\mu}$ can be determined from reduced system of linear equations (23) with any one (except the first one) of them eliminated with setting a corresponding multiplier equal to zero.

Putting the improved WSRD method to homothetic test, suppose that $\mathbf{q}=k \mathbf{e}_{N M}$. Inserting this formula into the orthogonality condition gives $(k-l) \mathbf{e}_{N M}^{\prime} \mathbf{W} \mathbf{e}_{N M}=0$ from which $l^{*}=k$. In turn, substituting the homothety into (22) leads to the homogeneous system

$$
\hat{\mathbf{a}}\left(\mathbf{G}^{\prime} \boldsymbol{\lambda}+\mathbf{H}^{\prime} \boldsymbol{\mu}\right)=\mathbf{0}_{N M}
$$

that obviously has the solution $\lambda^{*}=c \mathbf{e}_{N}, \boldsymbol{\mu}^{*}=-c \mathbf{e}_{M}$ where $c$ is an arbitrary scalar (see Sect. 6). Further, at $\mathbf{u}=k \mathbf{u}_{\mathrm{A}}, \mathbf{v}=k \mathbf{v}_{\mathbf{A}}$ and $l^{*}=k$ the linear equations (23) are also being transformed to the homogeneous system

$$
\begin{aligned}
& \mathbf{a}^{\prime}\left(\mathbf{G}^{\prime} \boldsymbol{\lambda}+\mathbf{a}^{\prime} \mathbf{H}^{\prime} \boldsymbol{\mu}\right)=0 \\
& \mathbf{G a ̂} \mathbf{W}^{-1} \hat{\mathbf{a}}\left(\mathbf{G}^{\prime} \boldsymbol{\lambda}+\mathbf{H}^{\prime} \boldsymbol{\mu}\right)=\mathbf{0}_{N} \\
& \mathbf{H a ̂} \mathbf{W}^{-1} \hat{\mathbf{a}}\left(\mathbf{G}^{\prime} \boldsymbol{\lambda}+\mathbf{H}^{\prime} \boldsymbol{\mu}\right)=\mathbf{0}_{M}
\end{aligned}
$$

with the same simple solution. Therefore, the vectors $\mathbf{q}^{*}=k \mathbf{e}_{N M}, \lambda^{*}=c \mathbf{e}_{N}, \boldsymbol{\mu}^{*}=-c \mathbf{e}_{M}$ bring the optimal solution to the problem of minimizing the objective function (18) subject to linear constraints (19) at $\mathbf{u}=k \mathbf{u}_{\mathrm{A}}$ and $\mathbf{v}=k \mathbf{v}_{\mathrm{A}}$.

Thus, the improved WSRD method passes through a homothetic test successfully as well as improved WSD method (and in contrast to WSRD method).

\section{Handling the zero entries in the distance minimization methods of matrix updating}

In practice, an initial matrix often contains some zero elements. In this context, all matrix updating methods can be divided into two groups: those that do preserve the same location of zeros in target matrix as in the initial one, and those that do not. For example, RAS method should be assigned to the first group because of its multiplicative pattern (3), whereas Kuroda's method belongs to the second group because it does not include any mechanisms for fitting the proper elements of target matrix to required zero level. Analogically, the regular and improved WSRD are methods of the first group 
because they are based on multiplicative pattern (16) in contradistinction to the WSD method both regular and improved.

If a zero preservation property is assumed desirable, the WSD methods can be slightly redeveloped to provide it by reducing the dimensionalities of minimization problems (8), (9) and (13), (9). Let $J<N M$ be a number of nonzero elements in the initial matrix $\mathbf{A}$ (and in $N M$-dimensional vector a). While the zero preservation principle "if $a_{j}=0$ then $x_{j}=0$ for any $j$ " is being applied, the target vector $\mathbf{x}$ does really contain only $J$ unknown variables because the other $N M-J$ variables should be setting to zero values. Hence, it is advisable to reduce the operational dimensions of initial and target vectors from $N M \times 1$ to $J \times 1$ by eliminating $N M-J$ zero components.

Let $\mathbf{E}_{J \times N M}$ be a rectangular matrix with dimensions $J \times N M$ that is obtained from an identity matrix of order NM by deleting the rows corresponding to zero entries in the initial vector. Then the initial and target vectors can be redefined as $\mathbf{a}_{J \times 1}=\mathbf{E}_{J \times N M} \mathbf{a}_{N M \times 1}$ and $\mathbf{x}_{J \times 1}=\mathbf{E}_{J \times N M} \mathbf{x}_{N M \times 1}$. In turn, in order to make the formulas of objective functions for WSD and improved WSD methods (8) and (13) be operational, one needs also to redefine the weight matrix $\mathbf{W}$ by deleting the corresponding rows and columns, i.e., $\mathbf{W}_{J \times J}=\mathbf{E}_{J \times N M} \mathbf{W}_{N M \times N M} \mathbf{E}_{J \times N M}^{\prime}$.

Finally, it is necessary to adapt the linear constraints (9) to reduced dimension of the target vector. To this end, $N \times N M$-dimensional matrix $\mathbf{G}$ and $M \times N M$-dimensional matrix $\mathbf{H}$ must be postmultiplied by $\mathbf{E}_{J \times N M}^{\prime}$ in order to eliminate their redundant columns. Hence, the constraints (9) are being transformed to $\mathbf{G}_{N \times N M} \mathbf{E}_{J \times N M}^{\prime} \mathbf{x}_{J \times 1}=\mathbf{u}_{N \times 1}$ and $\mathbf{H}_{M \times N M} \mathbf{E}_{J \times N M}^{\prime} \mathbf{x}_{J \times 1}=\mathbf{v}_{M \times 1}$, respectively, where all dimensions are consistent in a sense of matrix product. Notice that, as earlier, each column of the transformed constraint matrices includes exactly one nonzero (unit) element so that $\mathbf{e}_{N}^{\prime} \mathbf{G}_{N \times N M} \mathbf{E}_{J \times N M}^{\prime}=\mathbf{e}_{M}^{\prime} \mathbf{H}_{M \times N M} \mathbf{E}_{J \times N M}^{\prime}=\mathbf{e}_{J}^{\prime}$. Thus, the redevelopment of regular and improved WSD methods comes to proper reducing the dimensions of all vectors and matrices in the minimization problems (8), (9) and (13), (9) without their reformulation.

Furthermore, it is easy to see that Kuroda's method can be modified for preserving the zeros in a similar way.

As noted earlier, the regular and improved WSRD methods provide the same location of zeros in target matrix as in the initial one. Despite it, the eliminating procedure described above seems to be helpful for them also because the operation of excluding zero elements allows decreasing the dimension of solution space for the matrix-updating problem in significant degree. It is to be emphasized that macroeconomic matrices of high dimensions often appear to be very sparse in practice, up to more than $90 \%$ of zero elements. In such cases, the efficiency of computations within the minimization problems (17), (19) and (18), (19) increases rather noticeably.

\section{One more failure of homothetic testing: GRAS method}

The regular WSD and WSRD methods do not satisfy a homothetic test originally but can be improved by applying a homothetic paradigm. However, some methods of matrix updating demonstrate an incorrigible failure of homothetic testing. For instance, the generalized RAS (GRAS) method does not pass through a homothetic test and at the same time cannot be enhanced in this sense because of its nature. 
As it is well-known, "... RAS can only handle non-negative matrices, which limits its application to SUTs that often contain negative entries..."-see Temurshoev et al. (2011, p. 92). The GRAS method has been proposed by Junius and Oosterhaven (2003) for the initial matrices with some negative entries, and later it was redeveloped by Lenzen et al. (2007). (In order to be fair, it is appropriate to mention that a mathematical framework of GRAS method was described earlier by Günlük-Şenesen and Bates 1988.)

Any initial matrix $\mathbf{A}$ can be represented as $\mathbf{A}=\mathbf{P}-\mathbf{Q}$ where $\mathbf{P}$ is a matrix of positive entries and $\mathbf{Q}$ is a matrix containing the absolute values of negative entries. The GRAS method leans on the mixed additive-multiplicative pattern

$$
\mathbf{X}=\hat{\mathbf{r}} \mathbf{P} \hat{\mathbf{s}}-\hat{\mathbf{r}}^{-1} \mathbf{Q} \hat{\mathbf{s}}^{-1}
$$

where $\mathbf{r}$ and $\mathbf{s}$ are unknown $N \times 1$ and $M \times 1$ column vectors that are needed to be estimated subject to linear constraints (9). Notice that in the absence of negative entries GRAS method coincides with RAS method which is based on multiplicative pattern (3).

For homothetic testing the GRAS method, suppose that $\mathbf{X}=k \mathbf{A}=k \mathbf{P}-k \mathbf{Q}$. Inserting this homothety into the pattern (24) leads to the following pair of matrix equations:

$$
\hat{\mathbf{r}} \mathbf{P} \hat{\mathbf{s}}=k \mathbf{P}, \quad \hat{\mathbf{r}} \mathbf{Q} \hat{\mathbf{s}}=\frac{1}{k} \mathbf{Q} .
$$

The transition to Hadamard's products in the left-hand sides of these equations gives

$$
\left(\mathbf{r s}^{\prime}\right) \circ \mathbf{P}=k \mathbf{P}, \quad\left(\mathbf{r s}^{\prime}\right) \circ \mathbf{Q}=\frac{1}{k} \mathbf{Q}
$$

from which it follows that

$$
\mathbf{r s}^{\prime}=k \mathbf{e}_{N} \mathbf{e}_{M}^{\prime}, \quad \mathbf{r s} \mathbf{s}^{\prime}=\frac{1}{k} \mathbf{e}_{N} \mathbf{e}_{M}^{\prime}
$$

where $\mathbf{e}_{N} \mathbf{e}_{M}^{\prime}$ is outer product of two summation vectors, i.e., the matrix of dimension $N \times M$ with unit elements.

It is easy to see that the conditions (25) are met simultaneously if and only if $k= \pm 1$, or $\mathbf{X}= \pm \mathbf{A}$. Hence, the matrix homothety $\mathbf{X}=k \mathbf{A}=k \mathbf{P}-k \mathbf{Q}$ cannot be a feasible solution for GRAS method at all other values of $k(k \neq \pm 1)$. Thus, the GRAS method does not pass through a homothetic test.

The strict structure of mixed additive-multiplicative pattern (24) does not give any opportunity for improving the GRAS method on the base of homothetic paradigm without its considerable redevelopment. Here the main problem seems to be an inexact correspondence between the GRAS method's objective function and Kullback-Leibler divergence that Lemelin (2009) has rightly pointed out. Indeed, if initial matrix A contains some negative entries, then by virtue of the Kullback-Leibler probabilistic interpretation the nonlinear function (6) can be rewritten as

$$
\begin{gathered}
f_{K L}\left(\mathbf{X}^{+} / x^{+} ; \mathbf{A}^{+} / a^{+}\right)=\sum_{n=1}^{N} \sum_{m=1}^{M} \frac{\left|x_{n m}\right|}{x^{+}} \ln \left(\frac{a^{+}}{x^{+}} \frac{\left|x_{n m}\right|}{\left|a_{n m}\right|}\right) \\
=\frac{1}{x^{+}} \sum_{n=1}^{N} \sum_{m=1}^{M}\left|x_{n m}\right| \ln \left(\frac{\left|x_{n m}\right|}{\left|a_{n m}\right|}\right)+\ln \frac{a^{+}}{x^{+}}
\end{gathered}
$$


where $x^{+}=\mathbf{e}_{N}^{\prime} \mathbf{X}^{+} \mathbf{e}_{M}=\sum_{n=1}^{N} \sum_{m=1}^{M}\left|x_{i j}\right|$ and $a^{+}=\mathbf{e}_{N}^{\prime} \mathbf{A}^{+} \mathbf{e}_{M}=\sum_{n=1}^{N} \sum_{m=1}^{M}\left|a_{i j}\right|$. Notice that now a value of $x^{+} \neq \mathbf{e}_{N}^{\prime} \mathbf{u}=\mathbf{e}_{M}^{\prime} \mathbf{v}$ is not known a priori in contrast to the case (6) in which all initial matrix entries are implied to be nonnegative.

Factorizing the unknown variables in (26) as $\left|x_{n m}\right|=\left|a_{n m}\right| z_{n m}$, one can introduce the new variables $z_{n m}$ and then obtain the following objective function:

$$
f_{K L}\left(\mathbf{Z} ; \mathbf{A}^{+}\right)=\frac{\sum_{n=1}^{N} \sum_{m=1}^{M}\left|a_{n m}\right| z_{n m} \ln z_{n m}}{\sum_{n=1}^{N} \sum_{m=1}^{M}\left|a_{n m}\right| z_{n m}}-\ln \sum_{n=1}^{N} \sum_{m=1}^{M}\left|a_{n m}\right| z_{n m}+\ln \sum_{n=1}^{N} \sum_{m=1}^{M}\left|a_{n m}\right|
$$

that should be minimized subject to the linear marginal total constraints

$$
\sum_{m=1}^{M} a_{n m} z_{n m}=u_{n}, \quad n=1 \div N, \quad \sum_{n=1}^{N} a_{n m} z_{n m}=v_{m}, \quad m=1 \div M
$$

It is interesting to compare three summands in right-hand side of (27) with two summands of the GRAS method's objective function

$$
f\left(\mathbf{Z} ; \mathbf{A}^{+}\right)=\sum_{n=1}^{N} \sum_{m=1}^{M}\left|a_{n m}\right| z_{n m} \ln \frac{z_{n m}}{e}=\sum_{n=1}^{N} \sum_{m=1}^{M}\left|a_{n m}\right| z_{n m} \ln z_{n m}-\sum_{n=1}^{N} \sum_{m=1}^{M}\left|a_{n m}\right| z_{n m}
$$

that was being considered at Lenzen et al. (2007). Let a target matrix be equal to the initial matrix, i.e., $\mathbf{X}=\mathbf{A}$, from which it follows that $\mathbf{Z}=\mathbf{e}_{N} \mathbf{e}_{M}^{\prime}$. Then we have $f\left(\mathbf{e}_{N} \mathbf{e}_{M}^{\prime} ; \mathbf{A}^{+}\right)=-a^{+}$, whereas $f_{K L}\left(\mathbf{e}_{N} \mathbf{e}_{M}^{\prime} ; \mathbf{A}^{+}\right)=-\ln a^{+}+\ln a^{+}=0$. Therefore, the GRAS method's objective function (29) may take negative values, and an identity of indiscernibles is not being satisfied for it in contrast to function (27). Thus, there are no any reasons to consider (29) as a distance (or even quasi-distance) function.

Note that the first summand in right-hand side of (29) is just the original GRAS objective function that has been proposed by Junius and Oosterhaven (2003). It coincides with the numerator of first summand in (27) and, hence, cannot be considered as a measure of "information distance" from the viewpoint of dimensional analysis.

It is to be emphasized that nonlinear minimization problem (27), (28) is much more complicated computationally than the GRAS problem of minimizing the objective function (29) subject to constraints (28). The problem (27), (28) is likely to deserve a further analytical examination, although Lemelin (2009) has proposed to solve it numerically.

In turn, it is easy to see that in homothetic testing with $\mathbf{X}=k \mathbf{A}$ and $\mathbf{Z}=k \mathbf{e}_{N} \mathbf{e}_{M}^{\prime}$ the nonnegative objective function (27) reaches its absolute minimum value, since

$$
\begin{aligned}
f_{K L}\left(k \mathbf{e}_{N} \mathbf{e}_{M}^{\prime} ; \mathbf{A}^{+}\right) & =\frac{\sum_{n=1}^{N} \sum_{m=1}^{M}\left|a_{n m}\right| k \ln k}{\sum_{n=1}^{N} \sum_{m=1}^{M}\left|a_{n m}\right| k}-\ln \sum_{n=1}^{N} \sum_{m=1}^{M}\left|a_{n m}\right| k+\ln a^{+} \\
& =\ln k-\ln k a^{+}+\ln a^{+}=0 .
\end{aligned}
$$

It means that, from viewpoint of the Kullback-Leibler divergence minimization approach, the matrix homothety $\mathbf{X}=k \mathbf{A}$ can be classified as optimal solution for proportionality case of a general matrix-updating problem. Therefore, the modified GRAS 
(mGRAS) method based on the minimization problem (27), (28) passes through a homothetic test successfully.

\section{Numerical examples and concluding remarks}

Consider the Eurostat input-output data set given in "Box 14.2: RAS procedure" (see Eurostat, 2008, p. 452) for compiling some numerical examples. The $3 \times 4$-dimensional initial matrix A combines the entries in intersections of the columns "Agriculture," "Industry," "Services," "Final d." with the rows "Agriculture," "Industry," "Services" in "Table 1: Input-output data for year 0." Note that all the elements of this matrix are positive. The row marginal total vector $\mathbf{u}$ of dimension $3 \times 1$ is the proper part of the column "Output" in "Table 2: Input-output data for year 1," and the column marginal total vector $\mathbf{v}^{\prime}$ of dimension $1 \times 4$ involves the proper entries of the row "Total" in the near-mentioned data source.

Initial matrix $\mathbf{A}$ and target marginal totals $\mathbf{u}, \mathbf{v}^{\prime}$ are marked by italic font in the left half and in the right half of Table 1, respectively. The results of handling the data from Table 1 by RAS method with iterative processes (4) or (5) and by Kuroda's method (KM) of conditional minimizing the quadratic objective function (7) with $\mathbf{W}_{1}=\mathbf{W}_{2}=\mathbf{E}_{N M} / \mathbf{e}_{N M}^{\prime} \mathbf{E}_{N M} \mathbf{e}_{N M}$ subject to the linear marginal total constraints (9) are grouped in Table 2. The next two numerical examples demonstrate the results of applying the WSD and improved WSD methods as well as WSRD and improved WSRD methods for updating the available data set at $\mathbf{W}=\mathbf{E}_{N M} / \mathbf{e}_{N M}^{\prime} \mathbf{E}_{N M} \mathbf{e}_{N M}$ (see Tables 3 and 4, respectively). The calculated target matrices seem to be close among themselves.

Table 1 Initial matrix and the target marginal totals, Eurostat (2008), p. 452

\begin{tabular}{|c|c|c|c|c|c|c|c|c|c|c|c|}
\hline Year 0 & $A$ & & & & $u_{A}$ & Year 1 & $x$ & & & & $\mathbf{u}$ \\
\hline & 20.00 & 34.00 & 10.00 & 36.00 & 100.00 & & 19.16 & 33.38 & 10.14 & 32.10 & 94.78 \\
\hline & 20.00 & 152.00 & 40.00 & 188.00 & 400.00 & & 18.32 & 158.16 & 41.36 & 195.02 & 412.86 \\
\hline & 10.00 & 72.00 & 20.00 & 98.00 & 200.00 & & 9.80 & 76.48 & 22.08 & 104.32 & 212.68 \\
\hline $\mathbf{v}_{\mathrm{A}}^{\prime}$ & 50.00 & 258.00 & 70.00 & 322.00 & 700.00 & $\mathbf{v}^{\prime}$ & 47.28 & 268.02 & 73.58 & 331.44 & 720.32 \\
\hline
\end{tabular}

Table 2 The RAS and KM results for updating the data set from Table 1

\begin{tabular}{|c|c|c|c|c|c|c|c|c|c|c|c|}
\hline RAS & $x$ & & & & $u_{x}=u$ & $\mathrm{KM}$ & $x$ & & & & $\mathbf{u}_{\mathrm{x}}=\mathbf{u}$ \\
\hline & 17.94 & 32.77 & 9.76 & 34.31 & 94.78 & & 18.79 & 32.20 & 10.01 & 33.78 & 94.78 \\
\hline & 19.36 & 158.08 & 42.12 & 193.30 & 412.86 & & 18.91 & 158.41 & 42.18 & 193.35 & 412.86 \\
\hline & 9.98 & 77.17 & 21.70 & 103.84 & 212.68 & & 9.57 & 77.41 & 21.38 & 104.31 & 212.68 \\
\hline $\mathbf{v}_{\mathbf{X}}^{\prime}=\mathbf{v}^{\prime}$ & 47.28 & 268.02 & 73.58 & 331.44 & 720.32 & $\mathbf{v}_{\mathbf{X}}^{\prime}=\mathbf{v}^{\prime}$ & 47.28 & 268.02 & 73.58 & 331.44 & 720.32 \\
\hline
\end{tabular}

Table 3 The WSD and iWSD results for updating the data set from Table 1

\begin{tabular}{|c|c|c|c|c|c|c|c|c|c|c|c|}
\hline WSD & $\mathrm{X}$ & & & & $u_{x}=u$ & iWSD & $\mathrm{X}$ & & & & $u_{x}=u$ \\
\hline & 16.10 & 34.34 & 8.20 & 36.15 & 94.78 & & 17.40 & 33.68 & 8.91 & 34.80 & 94.78 \\
\hline & 20.62 & 156.86 & 42.72 & 192.67 & 412.86 & & 19.25 & 157.73 & 41.83 & 194.05 & 412.86 \\
\hline & 10.57 & 76.82 & 22.67 & 102.62 & 212.68 & & 10.63 & 76.62 & 22.85 & 102.59 & 212.68 \\
\hline $\mathbf{v}_{X}^{\prime}=\mathbf{v}^{\prime}$ & 47.28 & 268.02 & 73.58 & 331.44 & 720.32 & $\mathbf{v}_{\mathbf{X}}^{\prime}=\mathbf{v}^{\prime}$ & 47.28 & 268.02 & 73.58 & 331.44 & 720.32 \\
\hline
\end{tabular}


Table 4 The WSRD and iWSRD results for updating the data set from Table 1

\begin{tabular}{|c|c|c|c|c|c|c|c|c|c|c|c|}
\hline WSRD & $x$ & & & & $u_{x}=u$ & iWSRD & $x$ & & & & $u_{x}=u$ \\
\hline & 18.39 & 32.40 & 10.00 & 33.99 & 94.78 & & 18.35 & 32.41 & 10.03 & 33.99 & 94.78 \\
\hline & 19.06 & 158.84 & 42.66 & 192.29 & 412.86 & & 19.07 & 158.82 & 42.60 & 192.37 & 412.86 \\
\hline & 9.83 & 76.77 & 20.92 & 105.16 & 212.68 & & 9.86 & 76.79 & 20.95 & 105.08 & 212.68 \\
\hline $\mathbf{v}_{\mathbf{X}}^{\prime}=\mathbf{v}^{\prime}$ & 47.28 & 268.02 & 73.58 & 331.44 & 720.32 & $\mathbf{v}_{\mathbf{X}}^{\prime}=\mathbf{v}^{\prime}$ & 47.28 & 268.02 & 73.58 & 331.44 & 720.32 \\
\hline
\end{tabular}

The following numerical example is assigned to verify a response of WSD and WSRD methods to homothetic testing at $k=5$ (see Table 5). It is easy to see that the WSD target matrix does distinct from $5 \mathrm{~A}$ very significantly, whereas the difference between the WSRD target matrix and $5 \mathrm{~A}$ is noticeably less.

Furthermore, several numerical examples are intended for testing some methods' response to zero entries in the initial matrix. So let us disturb two elements of initial matrix $\mathbf{A}$ in Table 1 , say $(1,3)$ and $(2,1)$, by putting it equal to zero for years 0 and 1 . After recalculation of the marginal totals we get the data set in the left and right halves of Table 6. (Initial matrix $\mathbf{A}$ and target marginal totals $\mathbf{u}, \mathbf{v}^{\prime}$ are marked by italic font; zero entries are underlined.)

The left parts of Tables 7, 8 and 9 contain the target matrices calculated by methods that are not based on any multiplicative pattern, namely, WSD and improved WSD as well as Kuroda's method, respectively. It is easy to check that they do not really preserve the same location of zeros in target matrix as in the initial one. In contrast, the right parts of Tables 7, 8 and 9 present the results of additional applying the proposed procedure of reducing the dimension of solution space in the matrix updating problem described in Sect. 8 (with the letters "rd" after an abbreviation of method's title).

Other several numerical examples are intended to test some methods' responses to negative entries in the initial matrix. So let us disturb two elements of initial matrix A in Table 1 , say the same elements $(1,3)$ and $(2,1)$, by reversing their sign for years 0 and 1. After proper recalculation of the marginal totals, we obtain the data set presented in the left and right halves of Table 10. (Initial matrix $\mathbf{A}$ and target marginal totals $\mathbf{u}, \mathbf{v}^{\prime}$ are marked by italic font; negative matrix elements are underlined.)

The halves of Tables 11, 12 and 13 contain the target matrices calculated by methods that, in contrast to RAS, can be used in presence of negative entries, namely WSD and improved WSD methods, WSRD and improved WSRD methods, Kuroda's method and GRAS method, respectively. It is easy to see that they are all quite acceptable at small values of relative difference $(x-a) / a$; in particular, for the data set from Table 10 .

$$
(x-a) / a=\left(\mathbf{e}_{N}^{\prime} \mathbf{u}-\mathbf{e}_{N}^{\prime} \mathbf{u}_{\mathbf{A}}\right) / \mathbf{e}_{N}^{\prime} \mathbf{u}_{\mathbf{A}}=(663.4-640) / 640=3.7 \% .
$$

Table 5 The WSD and WSRD homothetic test results for the data set from Table 1 at $k=5$

\begin{tabular}{|c|c|c|c|c|c|c|c|c|c|}
\hline WSD & $X \neq 5 A$ & & & & $u_{x}=5 u_{A}$ WSRD & $X \neq 5 A$ & & & $u_{X}=5 u_{A}$ \\
\hline & -46.67 & 244.67 & $7-30.00$ & 332.00 & 500.00 & 127.17 & 166.38 & 175.28 & 500.00 \\
\hline & 253.33 & 662.67 & 300.00 & 784.00 & 2000.00 & 92.17 & 775.80238 .68 & 893.36 & 2000.00 \\
\hline & 43.33 & 382.67 & 80.00 & 494.00 & 1000.00 & 30.66 & $347.82 \quad 80.15$ & 541.36 & 1000.00 \\
\hline $\mathbf{v}_{\mathbf{X}}^{\prime}=5 \mathbf{v}_{\mathbf{A}}^{\prime}$ & 250.00 & 1290.00 & 350.00 & 1610.00 & 3500.00 & 250.00 & 1290.00350 .00 & 1610.00 & 3500.00 \\
\hline
\end{tabular}


Table 6 Initial matrix with zero entries and the target marginal totals

\begin{tabular}{|c|c|c|c|c|c|c|c|c|c|c|c|}
\hline Year 0 & A & & & & $u_{A}$ & Year 1 & $x$ & & & & u \\
\hline & 20.00 & 34.00 & $\underline{0.00}$ & 36.00 & 90.00 & & 19.16 & 33.38 & $\underline{0.00}$ & 32.10 & 84.64 \\
\hline & $\underline{0.00}$ & 152.00 & 40.00 & 188.00 & 380.00 & & $\underline{0.00}$ & 158.16 & 41.36 & 195.02 & 394.54 \\
\hline & 10.00 & 72.00 & 20.00 & 98.00 & 200.00 & & 9.80 & 76.48 & 22.08 & 104.32 & 212.68 \\
\hline $\mathbf{v}_{\mathbf{A}}^{\prime}$ & 30.00 & 258.00 & 60.00 & 322.00 & 670.00 & $\mathbf{v}_{\mathrm{A}}^{\prime}$ & 28.96 & 268.02 & 63.44 & 331.44 & 691.86 \\
\hline
\end{tabular}

Table 7 The WSD and WSDrd results for updating the data set from Table 6

\begin{tabular}{|c|c|c|c|c|c|c|c|c|c|c|c|}
\hline WSD & $x$ & & & & $u_{x}=u$ & WSDrd & $x$ & & & & $u_{x}=u$ \\
\hline & 16.49 & 34.18 & -2.02 & 35.99 & 84.64 & & 16.64 & 33.09 & $\underline{0.00}$ & 34.90 & 84.64 \\
\hline & 1.47 & 157.15 & 42.96 & 192.96 & 394.54 & & $\underline{0.00}$ & 158.16 & 42.42 & 193.97 & 394.54 \\
\hline & 11.00 & 76.69 & 22.50 & 102.50 & 212.68 & & 12.32 & 76.77 & 21.02 & 102.57 & 212.68 \\
\hline $\mathbf{v}_{\mathbf{X}}^{\prime}=\mathbf{v}^{\prime}$ & 28.96 & 268.02 & 63.44 & 331.44 & 691.86 & $\mathbf{v}_{\mathbf{X}}^{\prime}=\mathbf{v}^{\prime}$ & 28.96 & 268.02 & 63.44 & 331.44 & 691.86 \\
\hline
\end{tabular}

With the same location of underlined matrix elements as in Table 6

Table 8 The iWSD and iWSDrd results for updating the data set from Table 6

\begin{tabular}{|c|c|c|c|c|c|c|c|c|c|c|c|}
\hline iWSD & $\mathrm{X}$ & & & & $u_{x}=u$ & iWSDrd & $\mathrm{X}$ & & & & $\mathrm{u}_{\mathrm{x}}=\mathrm{u}$ \\
\hline & 18.13 & 33.47 & -1.51 & 34.55 & 84.64 & & 17.57 & 33.00 & $\underline{0.00}$ & 34.07 & 84.64 \\
\hline & -0.39 & 158.17 & 42.24 & 194.53 & 394.54 & & $\underline{0.00}$ & 158.37 & 41.44 & 194.74 & 394.54 \\
\hline & 11.22 & 76.38 & 22.72 & 102.36 & 212.68 & & 11.39 & 76.65 & 22.00 & 102.64 & 212.68 \\
\hline $\mathbf{v}_{\mathrm{X}}^{\prime}=\mathbf{v}^{\prime}$ & 28.96 & 268.02 & 63.44 & 331.44 & 691.86 & $\mathbf{v}_{\mathbf{X}}^{\prime}=\mathbf{v}^{\prime}$ & 28.96 & 268.02 & 63.44 & 331.44 & 691.86 \\
\hline
\end{tabular}

With the same location of underlined matrix elements as in Table 6

Table 9 The KM and KMrd results for updating the data set from Table 6

\begin{tabular}{|c|c|c|c|c|c|c|c|c|c|c|c|}
\hline KM & $X$ & & & & $u_{x}=u$ & KMrd & $x$ & & & & $u_{x}=u$ \\
\hline & 19.22 & 31.98 & $=0.15$ & 33.58 & 84.64 & & 19.23 & 31.91 & $\underline{0.00}$ & 33.51 & 84.64 \\
\hline & $\underline{0.02}$ & 158.62 & 42.28 & 193.62 & 394.54 & & $\underline{0.00}$ & 158.67 & 42.20 & 193.67 & 394.54 \\
\hline & 9.72 & 77.42 & 21.31 & 104.23 & 212.68 & & 9.73 & 77.45 & 21.24 & 104.26 & 212.68 \\
\hline $\mathbf{v}_{\mathbf{X}}^{\prime}=\mathbf{v}^{\prime}$ & 28.96 & 268.02 & 63.44 & 331.44 & 691.86 & $\mathbf{v}_{\mathrm{X}}^{\prime}=\mathbf{v}^{\prime}$ & 28.96 & 268.02 & 63.44 & 331.44 & 691.86 \\
\hline
\end{tabular}

Table 10 Initial matrix with negative entries and the target marginal totals

\begin{tabular}{|c|c|c|c|c|c|c|c|c|c|c|c|}
\hline Year 0 & A & & & & $u_{A}$ & Year 1 & $\mathrm{x}$ & & & & u \\
\hline & 20.00 & 34.00 & -10.00 & 36.00 & 80.00 & & 19.16 & 33.38 & $=10.14$ & 32.10 & 74.50 \\
\hline & -20.00 & 152.00 & 40.00 & 188.00 & 360.00 & & -18.32 & 158.16 & 41.36 & 195.02 & 376.22 \\
\hline & 10.00 & 72.00 & 20.00 & 98.00 & 200.00 & & 9.80 & 76.48 & 22.08 & 104.32 & 212.68 \\
\hline $\mathbf{v}_{\mathrm{A}}^{\prime}$ & 10.00 & 258.00 & 50.00 & 322.00 & 640.00 & $\mathbf{v}_{\mathrm{A}}^{\prime}$ & 10.64 & 268.02 & 53.30 & 331.44 & 663.40 \\
\hline
\end{tabular}

Setting the new target marginal totals, say, by doubling them, i.e., at

$$
(x-a) / a=\left(2 \mathbf{e}_{N}^{\prime} \mathbf{u}-\mathbf{e}_{N}^{\prime} \mathbf{u}_{\mathbf{A}}\right) / \mathbf{e}_{N}^{\prime} \mathbf{u}_{\mathbf{A}}=(1326.8-640) / 640=107.3 \%,
$$


Table 11 The WSD and iWSD results for updating the data set from Table 10

\begin{tabular}{|c|c|c|c|c|c|c|c|c|c|c|c|}
\hline WSD & $X$ & & & & $u_{x}=u$ & iWSD & $x$ & & & & $u_{x}=u$ \\
\hline & 16.89 & 34.02 & -12.23 & 35.82 & 74.50 & & 18.87 & 33.28 & -11.96 & 34.32 & 74.50 \\
\hline & -17.68 & 157.45 & 43.21 & 193.25 & 376.22 & & -20.05 & 158.60 & 42.68 & 194.99 & 376.22 \\
\hline & 11.43 & 76.56 & 22.32 & 102.37 & 212.68 & & 11.83 & 76.14 & 22.58 & 102.13 & 212.68 \\
\hline $\mathbf{v}_{\mathbf{x}}^{\prime}=\mathbf{v}^{\prime}$ & 10.64 & 268.02 & 53.30 & 331.44 & 663.40 & $\mathbf{v}_{\mathbf{X}}^{\prime}=\mathbf{v}^{\prime}$ & 10.64 & 268.02 & 53.30 & 331.44 & 663.40 \\
\hline
\end{tabular}

With the same location of underlined matrix elements as in Table 10

Table 12 The WSRD and iWSRD results for updating the data set from Table 10

\begin{tabular}{|c|c|c|c|c|c|c|c|c|c|c|c|}
\hline WSRD & $x$ & & & & $u_{x}=u$ & iWSRD & $x$ & & & & $u_{x}=u$ \\
\hline & 19.74 & 31.68 & -10.08 & 33.16 & 74.50 & & 19.97 & 31.71 & -10.37 & 33.20 & 74.50 \\
\hline & -19.32 & 159.67 & 42.52 & 193.35 & 376.22 & & -19.75 & 159.64 & 42.64 & 193.69 & 376.22 \\
\hline & 10.23 & 76.67 & 20.86 & 104.92 & 212.68 & & 10.42 & 76.68 & 21.04 & 104.55 & 212.68 \\
\hline $\mathbf{v}_{\mathbf{X}}^{\prime}=\mathbf{v}^{\prime}$ & 10.64 & 268.02 & 53.30 & 331.44 & 663.40 & $\mathbf{v}_{\mathbf{x}}^{\prime}=\mathbf{v}^{\prime}$ & 10.64 & 268.02 & 53.30 & 331.44 & 663.40 \\
\hline
\end{tabular}

With the same location of underlined matrix elements as in Table 10

Table 13 The KM and GRAS results for updating the data set from Table 10

\begin{tabular}{|c|c|c|c|c|c|c|c|c|c|c|c|}
\hline KM & $x$ & & & & $u_{x}=u$ & GRAS & $x$ & & & & $u_{x}=u$ \\
\hline & 21.23 & 31.13 & -10.58 & 32.72 & 74.50 & & 19.01 & 32.22 & -10.46 & 33.72 & 74.50 \\
\hline & -21.26 & 159.83 & 42.58 & 195.06 & 376.22 & & -19.08 & 158.88 & 42.19 & 194.23 & 376.22 \\
\hline & 10.67 & 77.06 & 21.30 & 103.65 & 212.68 & & 10.71 & 76.92 & 21.56 & 103.48 & 212.68 \\
\hline $\mathbf{v}_{\mathbf{x}}^{\prime}=\mathbf{v}^{\prime}$ & 10.64 & 268.02 & 53.30 & 331.44 & 663.40 & $\mathbf{v}_{\mathbf{X}}^{\prime}=\mathbf{v}^{\prime}$ & 10.64 & 268.02 & 53.30 & 331.44 & 663.40 \\
\hline
\end{tabular}

With the same location of underlined matrix elements as in Table 10

we obtain new target matrices calculated by the same methods; they are located in Tables 14, 15 and 16. It is to be emphasized that only the iWSD, iWSRD methods and Kuroda's method show acceptable results here, whereas the WSD, WSRD and GRAS methods are not good at all. Recall that exactly these methods do not satisfy a homothetic test proposed (see Sects. 5, 7 and 9, respectively).

The next numerical example is assigned to verify a response of GRAS method to homothetic testing at $k=2$ and $k=10$ (see Table 17). It is easy to see that the GRAS target matrices do differ from $2 \mathbf{A}$ and $10 \mathbf{A}$ very significantly. For instance, $2 a_{13}=-20$ and $2 a_{21}=-40$ whereas $x_{13}=-5.62$ and $x_{21}=-16.86$. In turn, at $k=10$ we have $10 a_{13}=-100$ and $10 a_{21}=-200$, whereas $x_{13}=-1.14$ and $x_{21}=-6.13$. Moreover,

Table 14 The WSD and iWSD results for the doubled marginal totals from Table 10

\begin{tabular}{|c|c|c|c|c|c|c|c|c|c|c|c|}
\hline WSD & $\mathrm{X}$ & & & & $u_{x}=2 u$ & iWSD & $\mathrm{X}$ & & & & $u_{x}=2 u$ \\
\hline & -16.22 & 86.70 & $=31.12$ & 109.64 & 149.00 & & 37.73 & 66.55 & $=23.92$ & 68.64 & 149.00 \\
\hline & $\underline{24.64}$ & 285.56 & 99.74 & 342.50 & 752.44 & & -40.11 & 317.21 & 85.36 & 389.98 & 752.44 \\
\hline & 12.87 & 163.79 & 37.97 & 210.73 & 425.36 & & 23.66 & 152.28 & 45.17 & 204.26 & 425.36 \\
\hline $\mathbf{v}_{\mathbf{x}}^{\prime}=2 \mathbf{v}^{\prime}$ & 21.28 & 536.04 & 106.60 & 662.88 & 1326.80 & $\mathbf{v}_{\mathbf{X}}^{\prime}=2 \mathbf{v}^{\prime}$ & 21.28 & 536.04 & 106.60 & 662.88 & 1326.80 \\
\hline
\end{tabular}


Table 15 The WSRD and iWSRD results for the doubled marginal totals from Table 10

\begin{tabular}{|c|c|c|c|c|c|c|c|c|c|c|c|}
\hline WSRD & $x$ & & & & $u_{x}=2 u$ & iWSRD & $x$ & & & & $u_{x}=2 u$ \\
\hline & 28.39 & 61.93 & -5.86 & 64.54 & 149.00 & & 39.94 & 63.41 & -20.75 & 66.40 & 149.00 \\
\hline & -18.35 & 320.93 & 79.30 & 370.57 & 752.44 & & -39.49 & 319.27 & 85.28 & 387.38 & 752.44 \\
\hline & 11.25 & 153.18 & 33.16 & 227.78 & 425.36 & & 20.83 & 153.35 & 42.07 & 209.10 & 425.36 \\
\hline $\mathbf{v}_{\mathbf{x}}^{\prime}=2 \mathbf{v}^{\prime}$ & 21.28 & 536.04 & 106.60 & 662.88 & 1326.80 & $\mathbf{v}_{\mathbf{x}}^{\prime}=2 \mathbf{v}^{\prime}$ & 21.28 & 536.04 & 106.60 & 662.88 & 1326.80 \\
\hline
\end{tabular}

Table 16 The KM and GRAS results for the doubled marginal totals from Table 10

\begin{tabular}{|c|c|c|c|c|c|c|c|c|c|c|c|}
\hline KM & $x$ & & & & $u_{x}=2 u$ & GRAS & $X$ & & & & $u_{x}=2 u$ \\
\hline & 42.46 & 62.26 & $=21.17$ & 65.45 & 149.00 & & 23.37 & 64.32 & $=5.94$ & 67.25 & 149.00 \\
\hline & $=42.51$ & 319.66 & 85.17 & 390.13 & 752.44 & & $=15.73$ & 312.83 & 73.26 & 382.07 & 752.44 \\
\hline & 21.33 & 154.12 & 42.60 & 207.31 & 425.36 & & 13.63 & 158.89 & 39.28 & 213.56 & 425.36 \\
\hline $\mathbf{v}_{\mathbf{X}}^{\prime}=2 \mathbf{v}^{\prime}$ & 21.28 & 536.04 & 106.60 & 662.88 & 1326.80 & $\mathbf{v}_{\mathbf{X}}^{\prime}=2 \mathbf{v}^{\prime}$ & 21.28 & 536.04 & 106.60 & 662.88 & 1326.80 \\
\hline
\end{tabular}

With the same location of underlined matrix elements as in Table 10

Table 17 The GRAS homothetic test results for the data set from Table 10 at $k=\mathbf{2}$ and 10

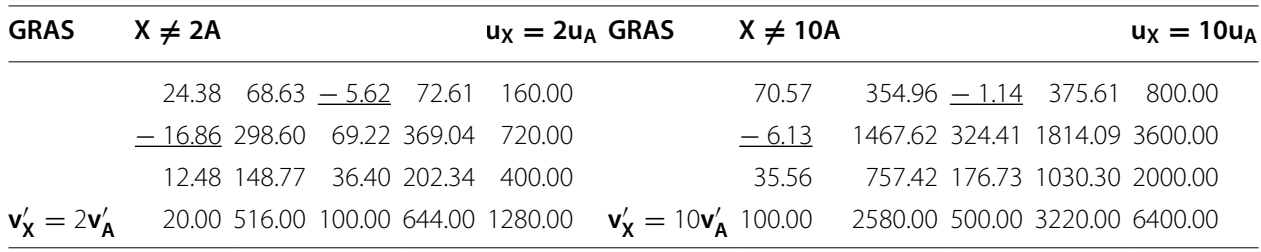

With the same location of underlined matrix elements as in Table 10

there are some other large distinctions between the matrix elements in the columns where the negative entries are located, namely, columns 1 and 3.

As a conclusion, it is to be emphasized that a homothetic paradigm expresses the important and helpful property of any matrix updating method. Moreover, positive response to homothetic testing serves as an additional evidence of plausibility and correctness of the matrix updating method tested.

Homothetic paradigm leans on a common notion of orthogonal projecting that is likely to be the most powerful concept in econometrics. It has an obvious logical interpretation, corresponds to famous principle of insufficient reason and remains operational in a row of practical situations.

If a method of matrix updating emanates from some distance minimization problem, it can be fully adapted to homothetic testing (see Sect. 6). If at the same time a zero preservation property is assumed desirable, the method must be slightly redeveloped to provide its observance, with a concomitant reducing the dimensionalities of underlying minimization problem without its reformulation (see Sect. 8).

In this article, we have considered four methods of matrix updating that can be used for handling the matrices with some negative entries, namely, Kuroda's, iWSD, iWSRD and GRAS (including mGRAS) methods. A critical analysis of the GRAS methodology is given in Sect. 9 (see also Tables 16 and 17). 
It is obvious that, because of its additive nature, the iWSD method does not work rather good if the initial matrix has some very small entries together with some very large ones (a practically important case!). The final numerical examples are intended to test a response of the above-mentioned methods to appearance of a small entry in the initial matrix. So let us disturb one element of initial matrix $\mathbf{A}$ in Table 10, say entry $(3,4)$, by multiplying its value by $10^{-4}$ for years 0 and 1 . After proper recalculation of the marginal totals, we obtain the data set presented in the left and right halves of Table 18. (Initial matrix $\mathbf{A}$ and target marginal totals $\mathbf{u}, \mathbf{v}^{\prime}$ are marked by italic font; the small elements $(3,4)$ are underlined.)

The results of handling the data from Table 18 by iWSD, Kuroda's and iWSRD methods are presented in Tables 19, 20 and 21 where the elements of matrices $\mathbf{Q}$ are determined by formula (16).

As it was expected, the iWSD method has unjustifiably reversed the sign of $a_{34}$ in target element $x_{34}$ and has increased the initial absolute value in more than 80 times although $x / a=\mathbf{e}_{N}^{\prime} \mathbf{u} / \mathbf{e}_{N}^{\prime} \mathbf{u}_{\mathrm{A}}$ is just equal to $559.09 / 542.01=1.03$ (see Table 19). In this situation Kuroda's method appears to work somewhat better without sign reversing and with increasing the initial value in more than only 10 times (see Table 20). And finally, the iWSRD method seems to be most preferable in this example demonstrating the scattering in Q's elements from 0.92 to 1.07 (see Table 21).

Table 18 Initial matrix with one small entry and the target marginal totals

\begin{tabular}{|c|c|c|c|c|c|c|c|c|c|c|c|}
\hline Year 0 & A & & & & $u_{A}$ & Year 1 & $x$ & & & & $\mathbf{u}$ \\
\hline & 20.00 & 34.00 & -10.00 & 36.00 & 80.00 & & 19.16 & 33.38 & -10.14 & 32.10 & 74.50 \\
\hline & -20.00 & 152.00 & 40.00 & 188.00 & 360.00 & & -18.32 & 158.16 & 41.36 & 195.02 & 376.22 \\
\hline & 10.00 & 72.00 & 20.00 & $\underline{0.01}$ & 102.01 & & 9.80 & 76.48 & 22.08 & $\underline{0.01}$ & 108.37 \\
\hline $\mathbf{v}_{\mathrm{A}}^{\prime}$ & 10.00 & 258.00 & 50.00 & 224.01 & 542.01 & $\mathbf{v}^{\prime}$ & 10.64 & 268.02 & 53.30 & 227.13 & 559.09 \\
\hline
\end{tabular}

Table 19 The iWSD results for updating the data set from Table 18

\begin{tabular}{|c|c|c|c|c|c|c|c|c|c|c|c|}
\hline iWSD & $X$ & & & & $u_{x}=u$ & iWSD & Q & & & & $\hat{u}_{A}^{-1} u_{X}$ \\
\hline & 18.94 & 33.56 & -11.75 & 33.75 & 74.50 & & 0.95 & 0.99 & 1.18 & 0.94 & 0.93 \\
\hline & -19.64 & 158.74 & 42.95 & 194.17 & 376.22 & & 0.98 & 1.04 & 1.07 & 1.03 & 1.05 \\
\hline & 11.34 & 75.71 & 22.11 & -0.79 & 108.37 & & 1.13 & 1.05 & 1.11 & -80.45 & 1.06 \\
\hline $\mathbf{v}_{\mathbf{X}}^{\prime}=\mathbf{v}^{\prime}$ & 10.64 & 268.02 & 53.30 & 227.13 & 559.09 & $\mathbf{v}_{\mathbf{X}}^{\prime} \hat{\mathbf{v}}_{\mathbf{A}}^{-1}$ & 1.06 & 1.04 & 1.07 & 1.01 & 1.03 \\
\hline
\end{tabular}

With the same location of underlined matrix elements as in Table 18

Table 20 The KM results for updating the data set from Table 18

\begin{tabular}{lrrrrrrrrrrr}
\hline KM & \multicolumn{1}{l}{$\mathbf{X}$} & & & & $\mathbf{u}_{\mathbf{X}}=\mathbf{u}$ & $\mathbf{K M}$ & $\mathbf{Q}$ & & & & $\hat{\mathbf{u}}_{\mathbf{A}}^{-1} \mathbf{u}_{\mathbf{X}}$ \\
\hline & 21.23 & 30.99 & -10.61 & 32.90 & 74.50 & & 1.06 & 0.91 & 1.06 & 0.91 & 0.93 \\
& -21.25 & 160.68 & 42.67 & 194.12 & 376.22 & & 1.06 & 1.06 & 1.07 & 1.03 & 1.05 \\
& 10.66 & 76.35 & 21.25 & $\underline{0.11}$ & 108.37 & & 1.07 & 1.06 & 1.06 & $\underline{10.82}$ & 1.06 \\
$\mathbf{v}_{\mathbf{X}}^{\prime}=\mathbf{v}^{\prime}$ & 10.64 & 268.02 & 53.30 & 227.13 & 559.09 & $\mathbf{v}_{\mathbf{X}}^{\prime} \hat{\mathbf{v}}_{\mathbf{A}}^{-1}$ & 1.06 & 1.04 & 1.07 & 1.01 & 1.03 \\
\hline
\end{tabular}

With the same location of underlined matrix elements as in Table 18 
Table 21 The iWSRD results for updating the data set from Table 18

\begin{tabular}{lrrrrrrrrrrr}
\hline iWSRD & \multicolumn{1}{l}{$\mathbf{X}$} & & \multicolumn{1}{c}{$\mathbf{u}_{\mathbf{X}}=\mathbf{u}$} & iWSRD & $\mathbf{Q}$ & & & & $\hat{\mathbf{u}}_{\mathbf{A}}^{-1} \mathbf{u}_{\mathbf{X}}$ \\
\hline & 19.90 & 31.69 & -10.28 & 33.20 & 74.50 & & 0.99 & 0.93 & 1.03 & 0.92 & 0.93 \\
& -19.62 & 159.34 & 42.58 & 193.92 & 376.22 & & 0.98 & 1.05 & 1.06 & 1.03 & 1.05 \\
& 10.37 & 76.99 & 21.00 & $\underline{0.01}$ & 108.37 & & 1.04 & 1.07 & 1.05 & $\underline{1.01}$ & 1.06 \\
$\mathbf{v}_{\mathbf{X}}^{\prime}=\mathbf{v}^{\prime}$ & 10.64 & 268.02 & 53.30 & 227.13 & 559.09 & $\mathbf{v}_{\mathbf{X}}^{\prime} \hat{\mathbf{v}}_{\mathbf{A}}^{-1}$ & 1.06 & 1.04 & 1.07 & 1.01 & 1.03 \\
\hline
\end{tabular}

With the same location of underlined matrix elements as in Table 18

Thus, one can assert that improved WSRD method has some advantage among the other methods of matrix updating in practically important situations when the initial matrix has some very small entries and some very large ones simultaneously (because of multiplicative nature of iWSRD objective function).

Notice that the minimization problems associated with Kuroda's and iWSRD methods have almost the same computational complexity. It can be shown that for their solving it is necessary to calculate the inverse of the symmetric matrix of order $\min \{N, M\}-1$ with the diagonal blocks on its main diagonal.

\section{Abbreviations}

KM: Kuroda's method; KMrd: Kuroda's method with a solution space of reduced dimension; WSD method: method of weighted squared differences; WSDrd: WSD method with a solution space of reduced dimension; iWSD method: improved WSD method; WSRD method: method of weighted squared relative differences; WSRDrd: WSRD method with a solution space of reduced dimension; iWSRD method: improved WSRD method; GRAS method: generalized RAS method; mGRAS method: modified GRAS method.

\section{Acknowledgements}

The author would like to thank anonymous referee and the participants of the International Conference on Economic Structures of PAPAIOS in Tokyo, Japan, March 17-19, 2017, and 19th National Conference of The Input-Output Research Association (IORA) of India in Pune, India, January 11-12, 2017, for their helpful comments and suggestions.

\section{Competing interests}

The author declares that he has no competing interests

Availability of data and materials

The data set supporting the conclusions of this article is available in Eurostat (2008, p. 452).

\section{Consent for publication}

Not applicable.

Ethics approval and consent to participate

Not applicable.

Funding

Not applicable.

\section{Publisher's Note}

Springer Nature remains neutral with regard to jurisdictional claims in published maps and institutional affiliations.

Received: 5 June 2017 Accepted: 5 October 2017

Published online: 22 November 2017

\section{References}

Eurostat (2008) European manual of supply, use and input-output tables. Methodologies and working papers. Office for Official Publications of the European Communities, Luxembourg

Günlük-Şenesen G, Bates JM (1988) Some experiments with methods of adjusting unbalanced data matrices. J R Stat Soc Ser A (Stat Soc) 151(3):473-490 
Harthoorn R, van Dalen J (1987) On the adjustment of tables with Lagrange multipliers. Occasional paper NA-024. The Netherlands Central Bureau of Statistics, National Accounts Research Division

Junius T, Oosterhaven J (2003) The solution of updating or regionalizing a matrix with both positive and negative entries. Econ Syst Res 15(1):87-96

Kullback S (1959) Information theory and statistics. Wiley, New York

Kullback S, Leibler RA (1951) On information and sufficiency. Ann Math Stat 22(1):79-86

Kuroda M (1988) A method of estimation for the updating transaction matrix in the input-output relationships. In: Uno K, Shishido S (eds) Statistical data bank systems. Socio-economic database and model building in Japan. North Holland, Amsterdam, pp 43-56

Lemelin A (2009) A GRAS variant solving for minimum information loss. Econ Syst Res 21(4):399-408

Lenzen M, Wood R, Gallego D (2007) Some comments on the GRAS method. Econ Syst Res 19(4):461-465

Magnus JR, Neudecker H (2007) Matrix differential calculus with applications in statistics and econometrics, 3rd edn. Wiley, Chichester

Miller RE, Blair PD (2009) Input-output analysis: Foundations and extensions, 2nd edn. Cambridge University Press, Cambridge

Temurshoev U, Webb C, Yamano N (2011) Projection of supply and use tables: methods and their empirical assessment. Econ Syst Res 23(1):91-123

\section{Submit your manuscript to a SpringerOpen ${ }^{\circ}$ journal and benefit from:}

- Convenient online submission

Rigorous peer review

Open access: articles freely available online

- High visibility within the field

Retaining the copyright to your article

Submit your next manuscript at $\boldsymbol{\Delta}$ springeropen.com 